\title{
Changes of perioperative hemoglobin and hematocrit levels in patients receiving total knee arthroplasty, a prospective observational study for an optimal timing of determination
}

\author{
Chenrong Ke \\ Wenzhou Medical University Second Affiliated Hospital \\ Naifeng Tian \\ Wenzhou Medical University Second Affiliated Hospital \\ Xiumeng Zhang \\ Nanjing Medical University Second Affiliated Hospital \\ Mochuan Chen ( $\nabla$ mochuan_chen11@sina.com ) \\ Wenzhou Medical University Second Affiliated Hospital
}

Research article

Keywords: total knee arthroplasty, blood loss, hemoglobin, hematocrit, transfusion

Posted Date: March 23rd, 2020

DOI: https://doi.org/10.21203/rs.3.rs-18062/v1

License: () This work is licensed under a Creative Commons Attribution 4.0 International License. Read Full License 


\section{Abstract}

Background Total knee arthroplasty (TKA) has long been associated with significant blood loss, hence postoperative hemoglobin $(\mathrm{Hb})$ and hematocrit $(\mathrm{Hct})$ determination remains necessary for evaluating blood loss and guiding transfusions. The aim of the study was to depict the changing patterns of perioperative $\mathrm{Hb}$ and Hct levels and to identify an optimal timing of determination in patients receiving TKA surgeries. Methods This was a prospective observational study by recruiting a total of 302 consecutive patients who received TKA surgeryfor final analysisfrom January 2017 until December 2018. Patients were kept in hospital for a full week postoperatively to detect a preoperative, postoperative day 1 through to day $7 \mathrm{Hb}$ and Hct concentrations, postoperative 1-month, 3-months and 6-monthsvalues were followed up in the clinic visiting. Results $\mathrm{Hb}$ and Hct levels decreased in the first few days and reached a nadir on postoperative day4 and day3 respectively while recovered in the following days and approached to preoperative values at postoperative 3months. The mean maximum drop of $\mathrm{Hb}$ and Hct concentrations was detected as $4.0 \mathrm{~g} / \mathrm{dL}$ and $11.5 \%$ separately. Significant differences were detected on $\mathrm{Hb}$ and $\mathrm{Hct}$ concentrations between the timing neighbors on preoperative vs. day1, day1 vs. day2, day2 vs. day3, day7 vs. 1 month, 1-monthvs. 3-months and Hct values at 3-months vs. 6-months. A total of 21 patients received blood transfusion perioperatively, among whom 4 patients were at postoperative day3and 5 patients were at day4. Conclusion $\mathrm{Hb}$ and Hct concentrations dramatically decreased during the first few days following TKA surgery whilegradually recovered from postoperative day4 and approached to the preoperative values at 3-months postoperatively. The changing patterns indicated an optimal timing of blood determination on postoperative day3 or day4 to accurately reflect ongoing hidden blood loss for better guiding blood transfusions.

\section{Background}

Total knee arthroplasty (TKA) is a successful, safe and cost-effective surgical procedure for end-staged knee arthritis to relieve pain, correct deformity and restore function [1, 2]. However, TKA has long been associated with significant drop on thehemoglobin $(\mathrm{Hb})$ and Hematocrit (Hct) levels because of perioperative visible and hidden blood loss, under which a blood transfusion is not uncommon in the early postoperative period thus rendering the patients at risks of transfusion reactions [3]. Several studies have highlighted the disadvantages of allogenic blood transfusion including a negative effect on postoperative complications, transfusion related issues, delayed length of hospital stay, higher cost and mortality [4-6]. Current researches on perioperative blood management during TKA surgery are mostly focused on rational application of tourniquet and drainage [7, 8], meticulous operative techniques [9], sealing of the intramedullary femoral canal [10] and prescription of tranexamic acid (TXA) or other agents [11]. Whereas, changing patterns of $\mathrm{Hb}$ and Hct levels after TKA surgery have rarely been to topic, and an optimal timing of hematological determination is still equivocal.

The fundamental target of blood management is to decrease the need for allogenic blood transfusion whilst at the same time preventing anemia. Blood transfusion trigger should be individualized by weighing the risks and benefits primarily based on the patients' postoperative $\mathrm{Hb}$ and Hct levels with associated medical comorbidities. Nevertheless, $\mathrm{Hb}$ thresholds used for determining whether a blood transfusion is requiredvary significantly across the literatures [12-14]. A systematic review [15] with ten trials included identified a transfusion trigger between 7.0 and $10.0 \mathrm{~g} / \mathrm{dL}$ (most often they were 8.0 or $9.0 \mathrm{~g} / \mathrm{dL}$ ); a recent study [16] addressing TKA surgery set a criteria for blood transfusion at a level of $\mathrm{Hb}<$ $8.0 \mathrm{~g} / \mathrm{dL}$ or symptomatic anemia at a level $<10.0 \mathrm{~g} / \mathrm{dL}$; current evidence[17] suggests the restrictive transfusion threshold using a lower $\mathrm{Hb}$ level to trigger transfusion (most commonly $7.0 \mathrm{~g} / \mathrm{dL}$ or $8.0 \mathrm{~g} / \mathrm{dL}$ ), and the liberal transfusion threshold using a higher $\mathrm{Hb}$ level to trigger transfusion (most commonly $9.0 \mathrm{~g} / \mathrm{dL}$ or $10.0 \mathrm{~g} / \mathrm{dL}$ ). Furthermore, preoperative $\mathrm{Hb}$ concentration is also regarded as a predictor for postoperative transfusion, Ryan and colleagues [18]indicated a preoperative $\mathrm{Hb}$ value of less than $12.5 \mathrm{~g} / \mathrm{dL}$ are particularly at risk and should be considered for blood conservation programs. 
Considering preoperative $\mathrm{Hb}$ concentration playing a significant role in the transfusion as well as a great variation in the postoperative $\mathrm{Hb}$ and $\mathrm{Hct}$ levels based on different timing of sample collections. We designed this prospective observational study:(1) to investigate the perioperative changes of $\mathrm{Hb}$ and Hct levels in patients receiving TKA surgeries; (2) to determine an optimal timing of sample collection for better guiding the practice of blood transfusions.

\section{Material And Methods Study design}

A prospective observational study investigating the perioperative changes of $\mathrm{Hb}$ and Hct values in addition to determinate the optimal timing of sample collection was conducted in patients receiving primary TKA surgery.In our department, patients were normally kept in hospital for a week after a TKA surgery, which made it feasible for blood collection all through to postoperative day7.The study was approved by the institutional ethic committee, and all recruited patients provided informed consent.

\section{Patients Selection}

All consecutive patients presenting between January 2017 until December 2018 with knee osteoarthritis thus receiving primary TKA surgeries and consenting to participate were prospectively screened. Inclusion criteria were: age over 55 years old; planning to receive unilateral TKA surgery; and preoperative $\mathrm{Hb}$ value higher than $12 \mathrm{~g} / \mathrm{dL}$ in male or $11 \mathrm{~g} / \mathrm{dL}$ in female. Patients were excluded from the study with the following conditions: disorders of the blood system such as coagulation deficiency, polycythemia or thrombocytopenia; a history of ipsilateral knee joint surgery. Patients were excluded in the following period as well if they receivean allogeneic blood transfusion or experienced significant complications.

\section{Perioperative Management}

Patients underwent the surgical procedure with the same general anesthetic protocol. Under tourniquet control, a standard TKA procedure was conducted through a midline incision and a medial parapatellar approach. All the surgeries were performed by one chief surgeon using a posterior cruciate-substituting cemented prosthesis (Genesis $₫$ Oxinium, Smith \& Nephew, Memphis, Tennessee, USA) in our department of joint division. $1 \mathrm{~g}$ of intravenous (IV) TXA was given prior to the skin incision, and drainage tube was normally inserted and removed at 24 hours after surgery.Postoperatively, all patients were mobilized according to a standardized physical therapy protocol under the guidance of doctors and nurses. Routine transfusion practice was conducted according to the local institutional policy with a Hb concentration lower than $7.0 \mathrm{~g} / \mathrm{dL}$ or lower than $9.0 \mathrm{~g} / \mathrm{dL}$ with symptomatic anemia.

\section{Data Assessment}

Demographic information including patients' age, gender, body mass index (BMI) as well as the perioperative values containing operation time, intraoperative blood loss and postoperative drainagevolume were collected and recorded. Levels of $\mathrm{Hb}$ and $\mathrm{Hct}$ were examined preoperatively after admission and on postoperative day 1 through to day7. Whether a patient received an allogenic blood transfusion at each time point was recorded in detail. Patients werefurther followed up at clinic visiting at 1 month, 3 months and 6 months postoperatively for screening their functional recovery and the $\mathrm{Hb}$ and Hct levels. 


\section{Statistical analysis}

Data was collected and recorded by using the Mircosoft Excel (Office 365, 2016 version) and analyzed by using standard statistical software (SPSS, version 22.0, Inc. USA). Continuous variables were presented as mean \pm standard deviation, of which the Student's t-test was used to investigate the differences between different time points. Results were considered as significant difference if the $p$ value was less than 0.05 .

\section{Results}

A total of 376 patients with primary unilateral TKAs in the prospective cohort were identified, of which 52 patients were excluded based on the inclusive and exclusive criteria. Perioperatively, 21 patients received allogenic blood transfusion according the institutional transfusion policy, and one patient experienced infection thus receiving a revision was excluded. Leaving a final of 302 patients recruited for this prospective observational study. A flow chart diagram was shown as Fig. 1.There were 104 male and 198 female patients with an average age of 71.2 years old. the preoperative $\mathrm{Hb}$ concentration was 13.4 (11.2 to 16.4) g/dL and Hct was 38.7 (29.6 to 47.1) \%. Demographic information was shown in Table 1.

Table 1

Demographic information of the patients

\begin{tabular}{|lllllllll|}
\hline Parameter & $\begin{array}{l}\text { Sex } \\
(\mathrm{M} / \mathrm{F})\end{array}$ & $\begin{array}{l}\text { Age (years } \\
\text { old })\end{array}$ & $\begin{array}{l}\mathrm{BMI} \\
\left(\mathrm{kg} / \mathrm{m}^{2}\right)\end{array}$ & $\begin{array}{l}\mathrm{Hb} \text { level } \\
(\mathrm{g} / \mathrm{dL})\end{array}$ & $\begin{array}{l}\text { Hct } \\
(\%)\end{array}$ & $\begin{array}{l}\text { Operation time } \\
(\mathrm{min})\end{array}$ & $\begin{array}{l}\text { Blood loss } \\
(\mathrm{ml})\end{array}$ & $\begin{array}{l}\text { Drainage } \\
(\mathbf{m l})\end{array}$ \\
\hline Value & $104 / 198$ & $71.2 \pm 8.6$ & $\begin{array}{l}26.7 \pm \\
2.9\end{array}$ & $13.4 \pm 1.6$ & $\begin{array}{l}38.7 \\
\pm 4.2\end{array}$ & $71.3 \pm 11.8$ & $212.6 \pm$ & $\begin{array}{l}287.5 \pm \\
49.8\end{array}$ \\
\hline
\end{tabular}

Changes of the $\mathrm{Hb}$ values during the follow-up time points were shown in Table 2 and Fig. $2 \mathrm{a}$. Hb values dramatically decreased in the early postoperative period and reached itsnadir at day4, thereafter began to increase on day5. At postoperative 3-months, the $\mathrm{Hb}$ value has already recovered to a $94.8 \%$ of the preoperative value, and almost reached to a similar value at postoperative 6-months. Significant differences were detected between the neighbor time points including preoperative vs. day1 ( $p<0.001)$; day1 vs. day2 $(p=0.018)$; day2 vs day3 $(p<0.001)$; day7 vs. 1-month $(p<$ $0.001)$; 1-month vs. 3-months ( $p<0.001)$; but no significant differences were found between other neighbors $(p>0.05)$.

Table 2

Changes of $\mathrm{Hb}$ values during the follow up time points

\begin{tabular}{|lllllllllllll|}
\hline $\begin{array}{l}\text { Time } \\
\text { point }\end{array}$ & Preoperative & Day1 & Day2 & Day3 & Day4 & Day5 & Day6 & Day7 & 1month & 3month & 6month \\
\hline Value & $13.4 \pm 1.6$ & 11.2 & 10.7 & $9.7 \pm$ & $9.4 \pm$ & $9.6 \pm$ & $9.9 \pm$ & 10.0 & $11.5 \pm$ & $12.7 \pm$ & $13.1 \pm$ \\
& & \pm 1.5 & \pm 1.3 & 1.5 & 1.3 & 1.4 & 1.6 & \pm 1.8 & 1.7 & 1.6 & 1.5 \\
\hline value & 0 & $2.2 \pm$ & $2.7 \pm$ & $3.7 \pm$ & $4.0 \pm$ & $3.8 \pm$ & $3.5 \pm$ & $3.4 \pm$ & $1.9 \pm$ & $0.7 \pm$ & $0.3 \pm$ \\
& & 0.4 & 0.5 & 0.6 & 0.5 & 0.6 & 0.4 & 0.6 & 0.4 & 0.6 & 0.6 \\
\hline
\end{tabular}

Changes of the Hct values during the follow-up time points were shown in Table 3 and Fig. $2 b$.Our results indicated that the Hct values were significantly correlated to the $\mathrm{Hb}$ concentration, which dramatically decreased in the first 3 days and reached a minimum at day3; thereafter, the Hct value fluctuated on day 4 and day 5 and began to increase on postoperative day5. At postoperative 3-months, the Hct concentration showed a delayed recovery to a $86.6 \%$ of preoperative value in comparison with $\mathrm{Hb}$ trends and also reached a similar value at 6-months postoperatively. Significant differences were detected between the neighbors on preoperative vs. day1 $(p<0.001)$; day1 vs. day2 ( $<$ 
$0.001)$; day2 vs day3 ( $\mathrm{p}=0.033)$; day7 vs. 1-month ( $<<0.001)$; 1-month vs. 3-months $(p<0.007)$ and 3-months vs. 6months $(p<0.001)$.

Table 3

Changes of Hct values during the follow up time points

\begin{tabular}{|llllllllllll|}
\hline $\begin{array}{l}\text { Time } \\
\text { point }\end{array}$ & Preoperative & Day1 & Day2 & Day3 & Day4 & Day5 & Day6 & Day7 & 1month & 3month & 6month \\
\hline Value & \multirow{2}{*}{$38.7 \pm 4.2$} & 32.6 & 29.3 & 27.2 & 28.4 & 27.8 & 29.1 & 29.5 & $31.2 \pm$ & $33.5 \pm$ & $36.6 \pm$ \\
& & \pm 4.8 & \pm 4.3 & \pm 5.1 & \pm 4.9 & \pm 5.2 & \pm 3.9 & \pm 4.4 & 6.3 & 4.9 & 5.6 \\
\hline \multirow{2}{*}{ value } & 0 & $6.1 \pm$ & $9.4 \pm$ & 11.5 & 10.3 & 10.9 & $9.6 \pm$ & $9.2 \pm$ & $7.5 \pm$ & $5.2 \pm$ & $2.1 \pm$ \\
& & 3.2 & 3.4 & \pm 3.7 & \pm 3.1 & \pm 3.6 & 3.4 & 3.9 & 3.3 & 3.7 & 3.4 \\
\hline
\end{tabular}

Perioperatively, a total of 21 patients who received allogenic blood transfusion, of whom3 patients had blood transfusion at an $\mathrm{Hb}$ value in the range of 7.0-9.0 g/dL with positive symptoms and others were based on a $\mathrm{Hb}$ value less than $7.0 \mathrm{~g} / \mathrm{dL}$. In regarding to thetiming of transfusion, 3 patients were during the operation, 3 patients were at postoperative day 1, 2 patients were at day2, 4 patients were at day3, 5 patients were at day 4 and the lastone was at day6. The results indicated that a lot more patients required a blood transfusion according to the postoperative day 3 and day $4 \mathrm{Hb}$ concentrations.

\section{Discussion}

TKA procedure is always companied with significant blood lossthus causing a postoperative anemia, understanding the changing patterns of postoperative blood variation is of particularly importancedue to the evidence between low $\mathrm{Hb}$ and the reduced capacity of rehabilitation $[19,20]$. In this study, we found that $\mathrm{Hb}$ and Hct levels dramatically decreased in the early postoperative periodand reached their nadirs at day 4 and day3 respectively, thereafter gradually recovered to preoperative values at 3 to 6-monthspostoperatively. Our results indicated an optimal timing of determination at postoperative day3 or day 4 to accurately reflect ongoing hidden blood loss for better guiding blood transfusions.

Total joint arthroplasty has historically been regarded as a surgical procedure that is amongst the most frequent products requiring blood transfusion. It was previously reported in 1988 that $50 \%$ of the TKA patients necessitating a blood transfusion perioperatively [21]. Over the past couple of decades, medical advances have greatly lowered the blood transfusions in patients undergoing these procedures with a transfusion rate varying from 2-19\% [22-24], which covered a comprehensive strategy of the preoperative, intraoperative and postoperative stages of a TKA surgery. Preoperatively, techniques have been focused on the supplementation with iron therapy [25], erythropoietin injection [26] and discontinuation of anticoagulants [27]. Intraoperative tactics include pharmacological treatment with anti-thrombolytic agents'prescription taken TXA for example [11], and non-pharmacological methods such as tourniquet use, hypotensive anesthesia, sealing of the intramedullary femoral canal, and shortening the operation time have demonstrated to be benefit [28]. Postoperatively, application of autologous transfusion drains and clamping of the surgical drains in good timing have decreased the need for transfusion [29.30].In our common practice of a TKA procedure, the above-mentioned blood salvage strategies were comprehensively engaged and a transfusion rate of $6.5 \%$ was received in our cohort. This number might have been lower as we monitored the $\mathrm{Hb}$ concentration at postoperative every day.

Researches focusing on the optimal timing of postoperative blood testing remains limited in the literature. As a matter of fact, TKA is associated with a significant hidden blood loss besides the visible volume. A study [31] focusing on postoperative lower limb swelling after TKA surgery indicated that swelling was most pronounced from the postoperative day3 to day5, which was most commonly representative of the ongoing blood loss thus the Hb level should be monitored during that period. Another study [32] addressing the hidden blood loss following TKA and found that this hidden blood 
loss represented $49 \%$ of the total measured blood loss. Recently, a retrospective cohort analysis with a total of 61 consecutive patients comparing the day 1 versus day $2 \mathrm{Hb}$ concentration after TKA and concluded that the day 2 blood testing is more likely to reflect the ongoing hidden blood loss [33]. In our present study, we prospectively observed the postoperative changes of $\mathrm{Hb}$ and Hct concentrations in a larger cohort and debated that the day3 or day4 blood testing might be an optimal timing of determination for reflecting hidden blood loss and better guiding blood transfusions.

Our findings are not without limitations. Firstly, the study was performed in a single institution with a relative number of Chinese patients which may not be generally globalized to other racial populations; secondly, the study was purely observational in design, under which a comparison was not able to be conducted; and lastly, our institutional transfusion policy for saving blood recourses at a $\mathrm{Hb}$ level of less than $7.0 \mathrm{~g} / \mathrm{dL}$ needs further validation to avoid misleading transfusion practice. Although a few limitations existed, the present study with a relative larger TKAs recognized the definite changing patterns of $\mathrm{Hb}$ and $\mathrm{Hct}$ concentrations following a TKA procedure, and hereby we proposed that a postoperative day3 or day4 might be the optimal timing of determination for better guiding blood transfusions.

\section{Conclusion}

Given the findings in the present study, the $\mathrm{Hb}$ and Hct concentrations dramatically decreased during the first postoperative 4 days following TKA while gradually recovered in the following and approached to the preoperative levels at 3-months postoperatively. The changing patterns indicated an optimal timing of $\mathrm{Hb}$ and Hct determination on postoperative day3 or day 4 to accurately reflect ongoing hidden blood loss for better guiding blood transfusions.

\section{Abbreviations}

TKA: total knee arthroplasty; Hb: hemoglobin; Hct: hematocrit; TXA: tranexamic acid.

\section{Declarations}

Ethics approval and consent to participate

The Ethics Committee of The Second Affiliated Hospital and Yuying Children's Hospital of Wenzhou Medical University approved the study protocol. Written informed consent was obtained from all patients enrolled in the investigation.

Consent for publication

Not applicable.

Availability of data and materials

The dataset supporting the conclusions of this article is available on request- please contact the corresponding author Dr. Mochuan Chen. Administrative permission was received from The Second Affiliated Hospital and Yuying Children's Hospital of Wenzhou Medical University to access the medical records.

Competing interests:

The authors declared that they have no Conflict of interests.

Funding:

Zhejiang Medical and Health Science and Technology Project (2017KY463)

Authors' contributions:

$\mathrm{CM}$ and $\mathrm{KC}$ designed the study and drafted the manuscript; CM, TN and ZX accumulated the data; TN and ZX analyzed the data; $\mathrm{CM}$ and $\mathrm{KC}$ proof the English language. All authors read and approved the final manuscript.

\section{References}


1.

Thienpont E, Schwab P, Fennema P. Efficacy of Patient-specific Instruments in Total Knee Arthroplasty: A Systematic Review and Meta-analysis[J]. J Bone Joint Surg Am. 2017;99(6):521-30.

2.

Zan P, Fan L, Liu K, et al. Reduction Osteotomy versus Extensive Release on Clinical Outcome Measures in Simultaneous Bilateral Total Knee Arthroplasty[J]. Med Sci Monit. 2017;23:3817-23.

3.

Lu Q, Peng H, Zhou GJ, et al. Perioperative Blood Management Strategies for Total Knee Arthroplasty[J]. Orthop Surg. 2018;10(1):8-16.

4.

Bernard AC, Davenport DL, Chang PK, et al. Intraoperative Transfusion of $1 \mathrm{U}$ to $2 \mathrm{U}$ Packed Red Blood Cells Is Associated with Increased 30-Day Mortality, Surgical-Site Infection, Pneumonia, and Sepsis in General Surgery Patients[J]. J Am Coll Surg. 2009;208(5):931-7.

5.

Frisch NB, Wessell NM, Charters MA, et al. Predictors and complications of blood transfusion in total hip and knee arthroplasty[J]. J Arthroplasty. 2014;29(9 Suppl):189-92.

6.

Hart A, Khalil JA, Carli A, et al. Blood transfusion in primary total hip and knee arthroplasty. Incidence, risk factors, and thirty-day complication rates[J]. J Bone Joint Surg Am. 2014;96(23):1945-51.

7.

Xu H, Xie J, Lei Y, et al. Closed suction drainage following routine primary total joint arthroplasty is associated with a higher transfusion rate and longer postoperative length of stay: a retrospective cohort study[J]. J Orthop Surg Res. 2019;14(1):163.

8.

Kim TK, Bamne AB, Sim JA, et al. Is lower tourniquet pressure during total knee arthroplasty effective? A prospective randomized controlled trial[J]. BMC Musculoskelet Disord. 2019;20(1):275.

9.

Watts CD. Pagnano MW:Minimising blood loss and transfusion in contemporary hip and knee arthroplasty[J]. J Bone Joint Surg Br. 2012;94(11 Suppl A):8-10.

10.

Li X, Qi X, Han X, et al. Effects of sealing the intramedullary femoral canal in total knee arthroplasty: A randomized study[J]. Med (Baltim). 2017;96(29):e7388.

11.

Shen PF, Hou WL, Chen JB, et al. Effectiveness and Safety of Tranexamic Acid for Total Knee Arthroplasty: A Prospective Randomized Controlled Trial[J]. Med Sci Monit. 2015;21:576-81.

12.

Mitchell MD, Betesh JS, Ahn J, et al. Transfusion Thresholds for Major Orthopedic Surgery: A Systematic Review and Meta-analysis[J]. J Arthroplasty. 2017;32(12):3815-21.

13.

Simon GI, Craswell A, Thom 0, et al:Impacts of Aging on Anemia Tolerance, Transfusion Thresholds, and Patient Blood Management[J].Transfus Med Rev, 2019; 5. pii: S0887-7963(18)30133-0.

14.

Laurén E, VikatmaaL,Kerkelä E, et al. Red blood cell transfusion in southern Finland from 2011 to 2016: a quality audit[J]. Transfus Med. 2019;29(1):41-7.

15. 
Carson JL, Hill S, Carless P, et al. Transfusion triggers: A systematic review of the literature[J]. Transfus Med Rev. 2002;16(3):187-99.

16.

Zan P, Mol MO, Yao JJ, et al. Release of the tourniquet immediately after the implantation of the components reduces the incidence of deep vein thrombosis after primary total knee arthroplasty[J]. Bone Joint Res. 2017;6(9):535-41.

17.

Carson JL, Stanworth SJ, Roubinian N, et al. Transfusion thresholds and other steategies for guiding allogeneic red blood cell transfusion[J]. Cochrane Database Syst Rev. 2016;10:CD002042.

18.

Ryan SP, Klement MR, Green CL, et al:Preoperative Hemoglobin Predicts Postoperative Transfusion Despite Antifibrinolytics During Total Knee Arthroplasty[J]Orthopedics, 2019; 42(2):103-9.

19.

Kim KI, Kim S, Kim KW, et al. Low hemoglobin A1C increases the risk of disability in community-dwelling older nondiabetics adults[J]. J Nutr Health Aging. 2016;20(3):341-6.

20.

Marinho CDL, Maioli MCP, Soares AR, et al. Predictive models of six-minute walking distance in adults with sickle cell anemia: Implications for rehabilitation[J]. J Bodyw Mov Ther. 2016;20(4):824-31.

21.

Bweman AT, Geissele AE, Bosacco SJ, et al: Blood Loss with Total Knee Arthroplasty[J]. Clin Orthop Relat Res, 1988; (234):137-138.

22.

Klika AK, Small TJ, Saleh A, et al. Primary Total Knee Arthroplasty Allogenic Transfusion Trends, Length of Stay, and Complications: Nationwide Inpatient Sample 2000-2009[J]. J Arthroplasty. 2014;29(11):2070-7.

23.

Bini SA, Darbinian JA, Brox WT, et al. Risk Factors for Reaching the Post-Operative Transfusion Trigger in a Community Primary Total Knee Arthroplasty Population[J]. J Arthroplasty. 2018;33(3):711-7.

24.

Yoshihara H, Yoneoka D. National Trends in the Utilization of Blood Transfusions in Total Hip and Knee Arthroplasty[J]. J Arthroplasty. 2014;29(10):1932-7.

25.

Petis SM, Lanting BA, Vasarhelyi EM, et al. Is There a Role for Preoperative Iron Supplementation in Patients Preparing for a Total Hip or Total Knee Arthroplasty?[J]. J Arthroplasty. 2017;32(9):2688-93.

26.

Karkouti K, Mccluskey SA, Evans L, et al. Erythropoietin is an effective clinical modality for reducing RBC transfusion in joint surgery[J]. Can J Anaesth. 2005;52(4):362-8.

27.

Meier R, Marthy R, Saely $\mathrm{CH}$, et al. Comparison of preoperative continuation and discontinuation of aspirin in patients undergoing total hip or knee arthroplasty[J]. Eur J Orthop Surg Traumatol. 2016;26(8):921-8.

28.

Banerjee S, Issa K, Kapadia B, et al. Intraoperative Nonpharmacotherapeutic Blood Management Strategies in Total Knee Arthroplasty[J]. J Knee Surg. 2013;26(6):387-93.

29.

Zan P, Yao JJ, Fan L, et al. Efficacy of a Four-Hour Drainage Clamping Technique in the Reduction of Blood Loss Following Total Hip Arthroplasty: A Prospective Cohort Study[J]. Med Sci Monit. 2017;23:2708-14.

30 . 
Markar SR, Jones GG, Karthikesalingam A, et al. Transfusion drains versus suction drains in total knee replacement: meta-analysis[J]. Knee Surg Sports Traumatol Arthrosc. 2012;20(9):1766-72.

31.

Gao FQ, Li ZJ, Zhang K, et al. Risk factors for lower limb swelling after primary total knee arthroplasty[J]. Chin Med J (Engl). 2011;124(23):3896-9.

32.

Sehat KR, Evans RL, Newman JH. Hidden blood loss following hip and knee arthroplasty. Correct management of blood loss should take hidden loss into account[J]. J Bone Joint Surg Br. 2004;86(4):561-5.

33.

Khalfaoui MY, Godavitarne C, Wilkinson MCP. Optimal Timing for Hemoglobin Concentration Determination after Total Knee Arthroplasty: Day 1 versus Day 2[J]. Knee Surg Relat Res. 2017;29(1):52-6.

\section{Figures}

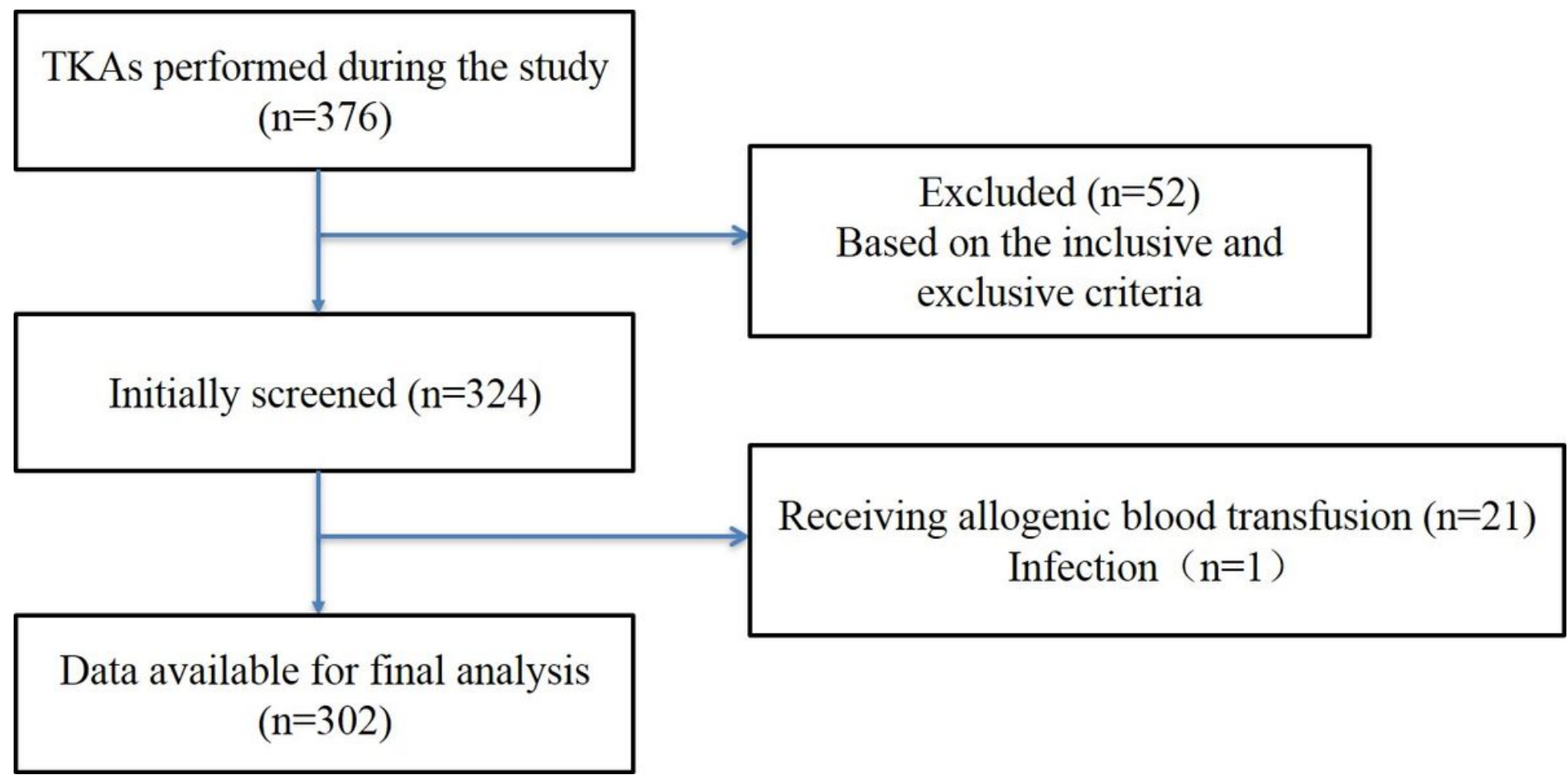

Figure 1

Patients' flow chart 
Changes of $\mathrm{Hb}$ levels with follow-up

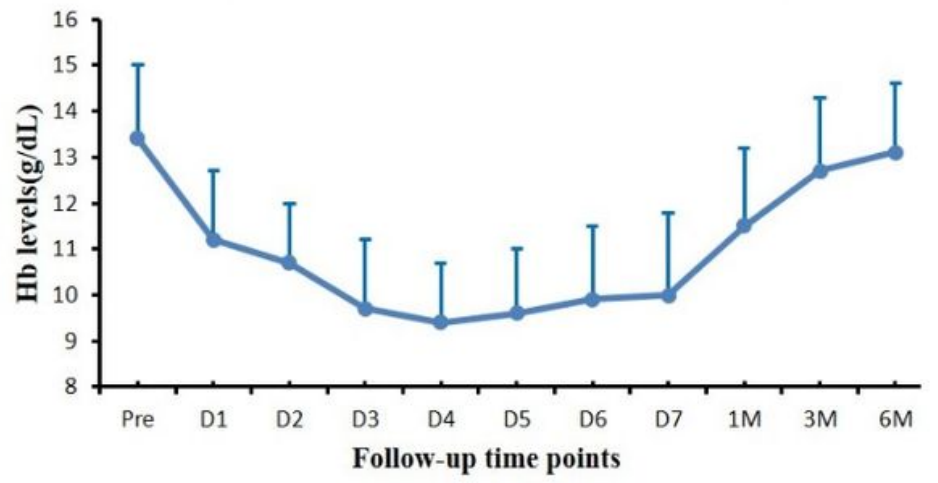

Changes of Het levels with follow-up

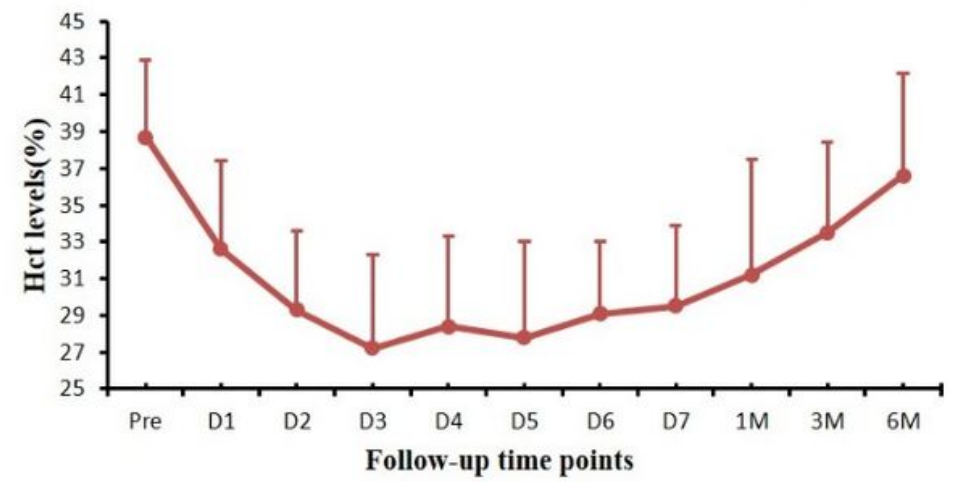

Figure 2

Changes of $\mathrm{Hb}$ and $\mathrm{Hct}$ levels with the follow-up time points ( $\mathrm{D}=$ day and $\mathrm{M}=$ month) 